\title{
Une décision essentielle en faveur des patients souffrant d'obésité
}

\section{Naef, F. Bauknecht, \\ A. Glättli, R. Hauser, \\ O. Huber, K. Laederach, \\ R. Peterli, M. Suter}

Swiss Society for the Study of Morbid Obesity and Metabolic Disorders (SMOB)

Traduction: M. Suter

Vous trouverez la version italienne sur le site Internet www.saez.ch $\rightarrow$ Numéro actuel ou $\rightarrow$ Archives $\rightarrow 2011 \rightarrow 3$

Correspondance:

Dr Markus Naef, MBA

Chirugische Klinik

Spital STS AG Thun

CH-3600 Thun

Tél. 0332262920

markus.naef@spitalstsag.ch
L'excès pondéral représente l'un des principaux problèmes de santé de la société moderne. Il touche environ 1,7 milliards de personnes, et l'on peut parler de véritable épidémie. En Suisse, 37,3\% de la population souffre d'un excès pondéral (30,3\% en 1992). Les comorbidités associées à l'obésité (hypertension artérielle, diabète, dyslipidémie, affections cardiovasculaires, etc.) constituent une charge socio-économique phénoménale. Les coûts engendrés par l'excès pondéral se montent en effet à près de 5,7 milliards de francs par année. En cas d'obésité sévère, les mesures conservatrices (régimes, médicaments, modifications comportementales) ne sont guère efficaces à long terme chez plus de $5 \%$ des sujets, et la chirurgie bariatrique représente le seul traitement efficace en ce qui concerne aussi bien la perte pondérale que l'amélioration des comorbidités et de la qualité de la vie. Ses effets se traduisent également par une diminution de la mortalité. De nombreuses études ont pu démontrer que la chirurgie de l'obésité représente une forme de traitement efficace, adéquate et économiquement saine $[1,2,3,4,5]$.

L'annexe 1 de l'ordonnance d'application de la LAMal définit les conditions dans lesquelles, en cas d'obésité, une intervention chirurgicale en vue d'une

\section{Tableau 1}

Modification de I'ordonnace d'application de la LAMal, annexe 1, chiffre 1.1 «Traitement chirurgical de l'adiposité».

\begin{tabular}{|c|c|}
\hline Mesures & Traitement chirurgical de l'adiposité \\
\hline Prise en charge & Oui \\
\hline \multirow[t]{5}{*}{ Conditions } & $\begin{array}{l}\text { 1. Le patient présente un indice de masse corporelle (IMC) supérieure } \\
\text { à } 35 \text {. }\end{array}$ \\
\hline & 2. Un traitement amaigrissant approprié de deux ans est resté sans effet. \\
\hline & $\begin{array}{l}\text { 3. Pose de l'indication, réalisation, assurance de la qualité et contrôle de } \\
\text { suivi, comme prévu dans le document «Richtlinien zur operativen } \\
\text { Behandlung von Übergewicht» du «Swiss Study Group for Morbid } \\
\text { Obesity» (SMOB) en date du } 9.11 .2010 \\
\text { (www.smob.ch, www.bag.admin.ch/ref). }\end{array}$ \\
\hline & $\begin{array}{l}\text { 4. Réalisation dans des centres qui, de par leur organisation et leur } \\
\text { personnel, sont en mesure de respecter les directives du SMOB du } \\
\text { 9.11.2010, dans le cadre du traitement chirurgical de l'adiposité. Les } \\
\text { centres certifiés par le SMOB sont réputés satisfaire à ces conditions. }\end{array}$ \\
\hline & $\begin{array}{l}\text { 5. Si l'intervention doit se dérouler dans un centre non certifié par } \\
\text { le SMOB, le médecin-conseil devra donner son accord préalable. }\end{array}$ \\
\hline Valable dès & 1.1 .2011 \\
\hline
\end{tabular}

perte de poids et de l'amélioration des comorbidités associés à l'obésité est à la charge de l'assurance maladie de base. Depuis sa fondation en 1996, le Swiss Society for the Study of Morbid Obesity and Metabolic Disorders (SMOB), en tant que groupe de spécialistes, représente l'organe compétent de dialogue avec la commission fédérale des prestations en ce qui concerne les recommandations de cette dernière

\section{Les patients dont le BMI est}

\section{$\geq 35 \mathrm{~kg} / \mathrm{m}^{2}$ peuvent bénéficier d'une intervention chirurgicale} sans demande préalable au médecin-conseil de la caisse-maladie concernée.

quant au traitement de l'obésité en Suisse. Le SMOB recommande depuis des années l'inclusion définitive de la chirurgie bariatrique dans le catalogue des prestations à la charge de l'assurance-maladie, également chez les malades dont l'indice de masse corporelle (Body Mass Index = BMI) est de $35-40 \mathrm{~kg} / \mathrm{m}^{2}$, ce qui constituerait une harmonisation des directives suisses avec les recommandations internationales établies. Le type d'intervention, quant à lui, doit être discuté entre le chirurgien, son équipe multidisciplinaire et le malade. Des demandes allant dans ce sens ont été déposées par le SMOB auprès de la commission fédérale des prestations le 31 mai 2006, puis à nouveau le 29 mai 2007. Ces demandes ont été soutenues par les impressionnantes données de la littérature internationale à disposition $[1,4,5,6,7]$. En vue d'une décision définitive en faveur de nos malades obèses, le SMOB a été chargé en date du 22 janvier 2008 d'établir des directives pour le traitement chirurgical de l'obésité, lesquelles serviraient de base à une modification de l'ordonnance d'application de la LAMal, dont les directives actuelles datent du 1 janvier 2000.

Le SMOB se réjouit, avec tous les partenaires concernés, que dans l'établissement de la nouvelle version de l'ordonnance d'application de la LAMal, qui entrera en vigueur le 1 janvier 2011, pratiquement toutes les demandes du SMOB aient été prises 


\begin{abstract}
Tableau 2
Conditions requises pour les centres bariatriques primaires.

\section{Centres bariatriques primaires}

Interventions autorisées: uniquement interventions standard: gastroplastie verticale, cerclage gastrique, bypass gastrique proximal sur anse en $Y$ (anse alimentaire $\leq 150 \mathrm{~cm}$ )

Interventions uniquement chez des malades dont le BMl est $<50 \mathrm{~kg} / \mathrm{m}^{2}$

Pas d'interventions chez l'enfant/adolescent ( $<18$ ans révolus)

Pas d'intervention chez les malades dont l'age est $>65$ ans révolus

Pas de réinterventions

Le chirurgien responsable du programme doit avoir une expérience d'au moins deux ans en chirurgie bariatrique.

Le chirurgien responsable du programme doit avoir réalisé au minimum 50 interventions bariatriques.

Nombre minimal d'interventions par année: 25 opérations

[12 interventions par année (1 chirurgien) suffisent durant la période de transition du 1.1.2011 au 31.12.2012].

Dans l'optique d'une stratégie de réseau, la collaboration avec une centre de référence en vue de la réalisation d'autres interventions est recommandée.
\end{abstract}

\section{Tableau 3}

Conditions requises pour les centres bariatriques de référence.

\section{Centres bariatriques de référence}

Interventions autorisées: toutes, c'est-à-dire gastroplastie verticale, cerclage gastrique, bypass gastrique proximal sur anse en $Y$ (anse alimentaire $\leq 150 \mathrm{~cm}$ ), dérivation bilio-pancéatique avec ou sans switch duodénal, sleeve gastrectomy, interventions en deux temps (première intervention: sleeve gastrectomy, seconde intervention: bypass gastrique proximal ou switch duodénal), bypass gastrique distal (canal commun $\leq 100 \mathrm{~cm}$ )

Interventions chez les malades à haut risque (BMI $\left.\geq 50 \mathrm{~kg} / \mathrm{m}^{2}\right)$

Réinterventions

Interventions chez les enfants/adolescents ( $<18$ ans révolus)

Interventions chez les malades dont l'âge est $>65$ ans révolus

Le chirurgien responsable du programme doit avoir une expérience d'au moins 5 ans en chirurgie bariatrique.

Le chirurgien responsable du programme doit avoir réalisé au minimum 200 interventions bariatriques.

Nombre minimum d'interventions par année: 50

[20 interventions ( 1 chirurgien), respectivement 30 interventions ( 2 chirurgiens) suffisent durant la période de transition du 1.1.2011 au 31.12.2012].

Dans l'optique d'une stratégie en réseau, la collaboration avec un ou plusieurs centres primaires est recommandée. mande préalable au médecin-conseil de la caissemaladie concernée. D'autre part, la notion d'une casuistique minimale est pour la première fois introduite pour les centres qui pratiquent ces opérations. Les directives du SMOB déterminent notamment que les méthodes d'évaluation et de prise en charge des malades doivent être standardisées dans les différents centres, et que ces derniers doivent se soumettre à un contrôle de qualité constant. Deux types de centres bariatriques sont définis, les centres bariatriques primaires et les centres bariatriques de référence. La liste des centres individuels qui remplissent toutes les conditions contenues dans les directives du SMOB, et qui par conséquent sont autorisés à facturer leurs prestations bariatriques à l'assurance-maladie de base, est publiée par l'Office Fédéral de la Santé via un lien Internet (Registre Chirurgie Bariatrique, www.bag. admin.ch). Les conditions auxquelles doivent répondre les deux types de centres sont exprimées dans les tableaux 2 et 3 .

L'excès pondéral et les comorbidités qui lui sont associées vont continuer, dans le futur, à occuper une place importante tant dans la politique de la santé que sur le plan socio-économique. La problématique est complexe et ne peut être abordée que dans le cadre de discussions communes et interdisciplinaires. Le SMOB se réjouit en ce qui concerne les importants progrès déjà faits en faveur de nos malades, et se tient volontiers à disposition en vue de futures discussions ouvertes et constructives.

\section{Références}

1 Sjöström L, Lindroos AK, Peltonen M et al. Lifestyle, diabetes and cardiovascular risk factors 10 years after bariatric surgery. N Engl J Med. 2004;351:2683-93.

2 Christou NV, Sampalis JS, Liberman M et al. Surgery decreases long-term mortality, morbidity and health-care use in morbidly obese patients. Ann Surg. 2004;240:416-24.

3 Buchwald H. Bariatric surgery for morbid obesity: health implications for patients, health professionals and third-party payers. 2004 ASBS consensus conference statement. Surg Obes Rel Dis. 2005;1: 371-81.

4 Suter M, Paroz A, Calmes JM et al. European experience with laparoscopic Roux-en-Y gastric bypass in 466 obese patients. Br J Surg. 2006;93: 726-32.

5 Naef M, Mouton WG, Naef U et al. Graft survival and complications after laparoscopic gastric banding for morbid obesity - Lessons learned from a 12-year experience. Obes Surg. 2010;20:1206-14.

6 Fried M, Hainer V, Basdevant A et al. Inter-disciplinary European guidelines on surgery of severe obesity. Int J Surg. 2007;1:1-9.

7 SAGES Guidelines Committee. SAGES guideline for clinical application of laparoscopic bariatric surgery. Surg Endosc. 2008;22:2281-300. dont le BMI est $\geq 35 \mathrm{~kg} / \mathrm{m}^{2}$ peuvent, sans limite d'âge, bénéficier d'une intervention chirurgicale sans de- en compte. En effet, la décision, signée par le chef le traitement chirurgical de l'obésité (valables dès le 9 novembre 2010 et accessibles via www.smob.ch ou www.bag.admin.ch/ref), est déterminante pour traitement de l'excès pondéral et pour la chirurgie de l'obésité en Suisse (tabl. 1). Deux modifications principales sont ainsi introduites. D'une part, les patients 